\title{
PRENATAL EXPOSURE TO OPIOID MAINTENANCE TREATMENT (OMT) REDUCES COGNITIVE PERFORMANCE IN YOUNG ADULT RATS
}

Mette Kongstorp ${ }^{1,2}$, Inger Lise Bogen ${ }^{1,2}$, Tom Stiris ${ }^{2,3}$, Jannike Mørch Andersen ${ }^{1,4}$

1) Department of Forensic Medicine, Oslo University Hospital, Oslo, Norway, 2) Faculty of Medicine, University of Oslo, Oslo, Norway,

3) Department of Neonatal Intensive Care, Oslo University Hospital, Oslo, Norway, 4) School of Pharmacy, University of Oslo, Oslo, Norway

\section{BACKGROUND}

Opioid maintenance treatment (OMT) is the recommended therapy for heroin addicted individuals, including pregnant women. However, the current knowledge concerning possible negative long-term effects on cognitive performance in children born by mothers in OMT is both limited and conflicting. In human studies, it might be difficult to separate effects of a drug from genetic, social and environmental factors. Hence, in this study we used an animal model, mimicking clinical exposure to OMT, to examine possible effects on learning and memory in young adult rats prenatally exposed to methadone or buprenorphine.

\section{METHODS}

Female rats were implanted with a 28-days osmotic minipump (Alzet, Cupertino, CA), delivering methadone (10 mg/kg/day), buprenorphine $(1 \mathrm{mg} / \mathrm{kg} / \mathrm{day})$ or sterile water, 5 days before mating. Once the offspring reached young adult age, two different behavior tests were performed to examine possible long-term effects on learning and memory. At 7 weeks of age, the rats underwent the Simultaneous brightness discrimination (SBD) test [1], which reflects non-spatial reference learning and memory. The animals were deprived of water during testing and were trained to discriminate between three interchangeable cylinders, of which one was filled with water. The position of the water-filled cylinder was changed between the trials in a prearranged randomized order. To succeed, five correct responses in succession were required during both learning (day 2) and memory (day 15) testing. At 10 weeks of age, the offspring were tested in the Morris water maze (MWM) [2], which examines spatial learning and memory. During learning (day $1-4$ ), the rats were trained to find a hidden platform placed in the north east (NE) quadrant of the pool, to escape from the water. For the memory (day 5 and 12) tests, the platform was removed and the animals were allowed to swim in the pool for 30 sec. Time spent in the NE quadrant was recorded.

RESULTS
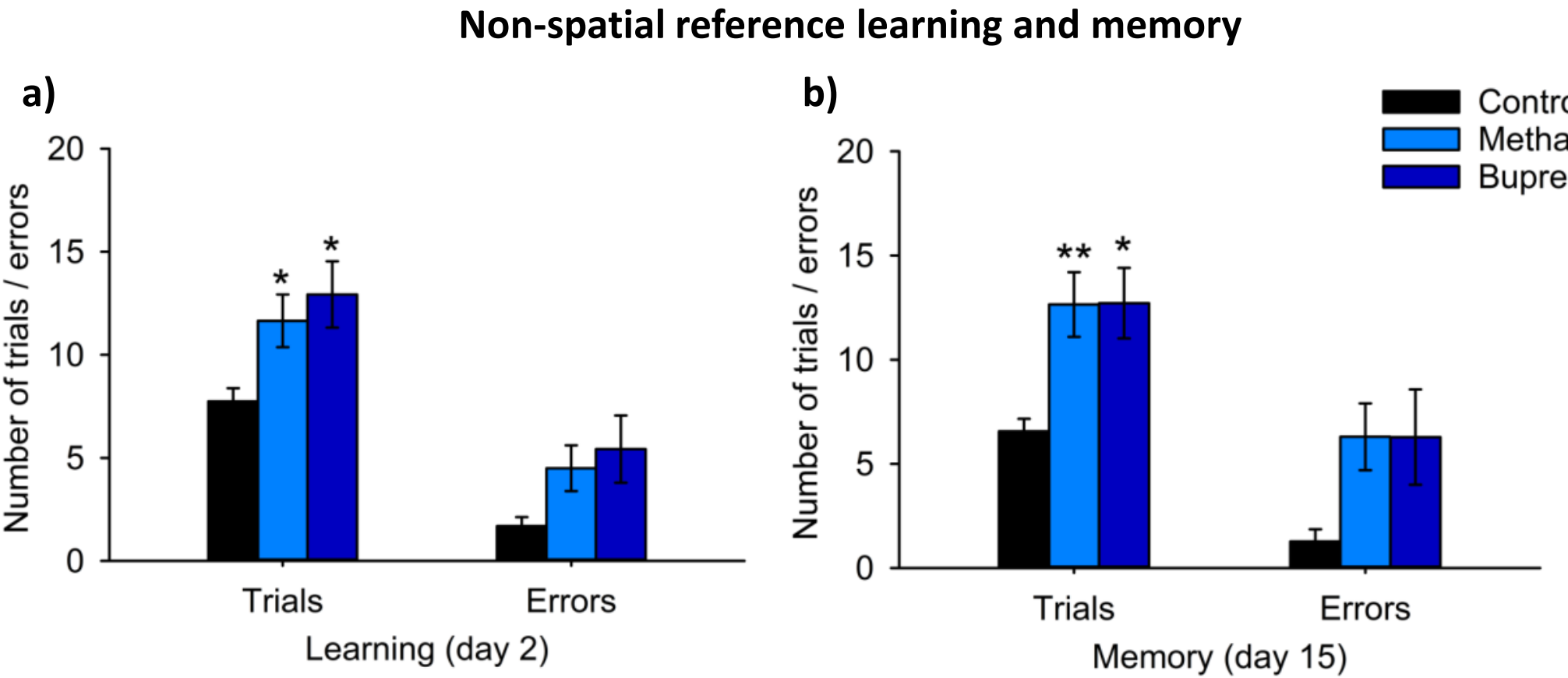

Figure 1. SBD test. Number of trials and errors used to achieve 5 correct responses in succession during the a) learning and b) memory test. c) Schematic overview of the test. The water-filled cylinder is marked in blue. Results are shown as mean \pm SEM. $n=7-10$ litters, $* p<0.05, * * p<0.01$.

\section{Spatial learning and memory}

a)

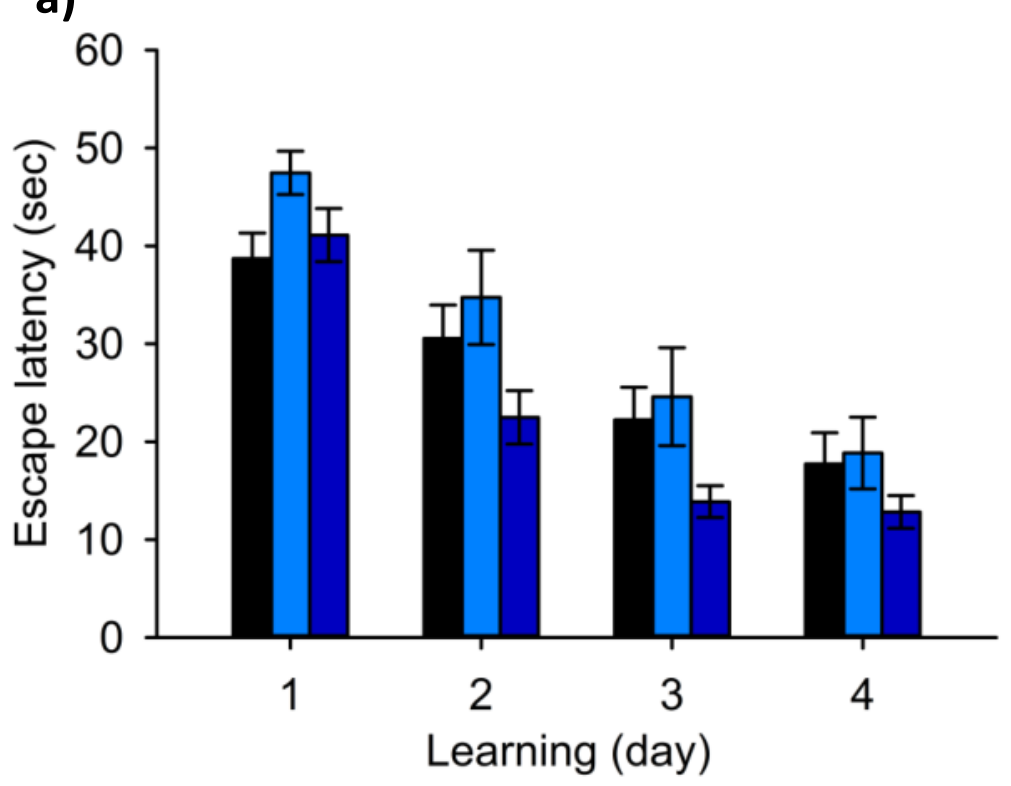

b)

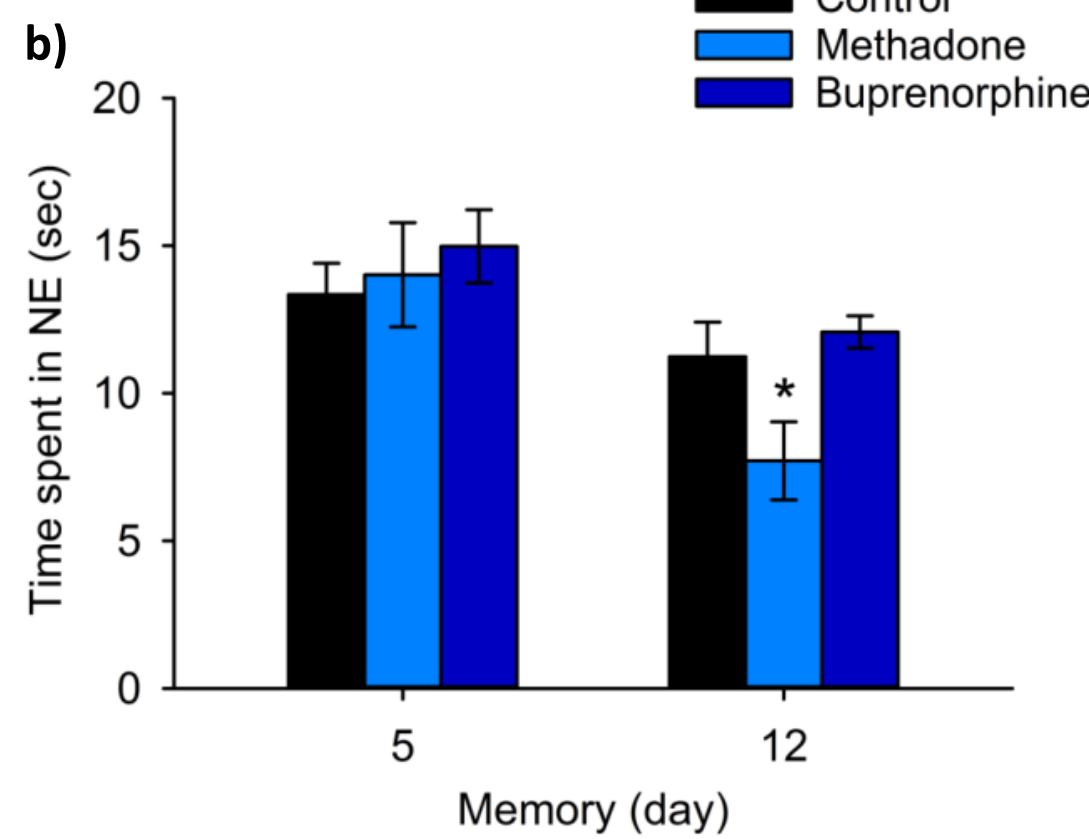

Figure 2. MWM test. a) Time used to find the hidden platform in the NE quadrant during learning. b) Time spent in the NE quadrant during the memory tests when the platform was removed. c) Schematic overview of the test. Results are shown as mean \pm SEM. $n=10$ litters, ${ }^{*} p<0.05$. c)

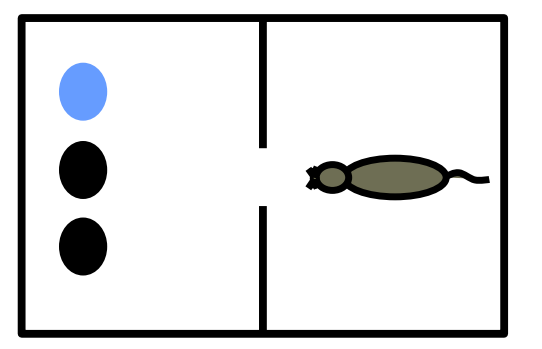

- At 7 weeks of age the opioid exposed offspring used a significantly higher number of trials to achieve the learning criterion during both the learning and memory tests compared to nonexposed rats. Opioid exposed rats also showed a tendency to make more mistakes $(p=0.06)$.

\section{CONCLUSION}

Offspring prenatally exposed to methadone or buprenorphine exhibited reduced non-spatial reference learning and memory. Methadone exposed offspring also displayed reduced long-term spatial memory. These findings show that exposure to clinical relevant methadone or buprenorphine concentrations during early development causes impaired cognitive performance in young adult rats. This is of great concern given the rapid increase in opioid use among fertile women. 